Pacific Journal of Mathematics

SUMMABILITY AND FOURIER ANALYSIS 


\title{
SUMMABILITY AND FOURIER ANALYSIS
}

\author{
George Brauer
}

An integration on $\beta N$, the Stone-Cech compactification of the natural numbers $N$, is defined such that if $s$ is a bounded sequence and $\dot{\rho}$ is a summation method evaluating $s$ to $\sigma$, $\int s d \dot{\phi}=\sigma$. The Fourier transform $\dot{\phi}$ of a summation method $\phi$ is defined as a linear functional on a space of test functions analytic in the unit disc: if

$$
f(z)=\sum_{n=0}^{\infty} \hat{f}(n) z^{n},|z|<1, \text { then } \phi(f)=\int \hat{f}(n) d \dot{\phi} .
$$

A functional which agrees with the Fourier transform of a regular summation method must annihilate the Hardy space $H_{1}$. Our space of test functions is often the space $M_{p}$ of functions $f=\Sigma \hat{f}(n) z^{n}$, analytic in the unit disc, such that

$$
\|f\|_{M_{p}}=\lim \sup \left[(1-r) \int_{0}^{2 \pi}\left|f\left(r^{1^{\prime} p^{\prime}} e^{i \theta}\right)\right|^{p} d \theta / 2 \pi\right]^{1 / p}
$$

is finite for some $p>1$. A functional $L$ which is well defined on a space $M_{p}$ for some $p \geqq 2$ such that $L(1 /(1-z))=1$ agrees with the Fourier transform of a summation method which is slightly stronger than convergence.

Let $s=\left\{s_{u}\right\}$ be an infinite sequence of complex numbers, that is, a continuous function on the discrete additive semigroup of natural numbers $N$. The sequence $s$ has a continuous extension $s^{\beta}$ to $\beta N$, the Stone-Cech compactification of $N\left(s^{3}\right.$ takes the value if $s$ is unbounded). Throughout the paper, the symbol $\beta Z$ denotes the Stone-Cech compactification of the space $Z$, and the continuous extension of a function $f$ defined on $Z$ to $\beta Z$ will be denoted by $f^{\beta}$; for a description of the Stone-Cech compactification we refer the reader to [2, pp. 82-93]. We impose the norm

$$
\begin{aligned}
\|s\| & =\lim \sup \left|s_{n}\right| \\
& =L U B \mid s^{\beta}(\gamma), \quad \gamma \in \beta N-N
\end{aligned}
$$

on the space $m_{0}$ of bounded sequences. Thus $m_{0}$ is isometric to $C(\beta N-N)$, the ring of continuous complex functions on $\beta N-N$; sequences differing by a null sequence are identified in $m_{n}$.

Let $\dot{s}$ denote a summation method-that is, a linear functional on a subspace of $m_{n}$. We assume that the $\dot{p}$-transform of every sequence $s$ to which $\dot{q}$ is applicable is either a continuous function on $N$ or else a continuous function on the half open unit interval $I$ : $[0,1)$. 
For example, if $\phi$ is representable by a summation matrix $A=\left(a_{n k}\right)$, then the $\phi$-transform of a sequence $s$ is the sequence $t$ given by

$$
t_{n}=\sum_{k=0}^{\infty} a_{n k} s_{k} \quad n=0,1, \cdots,
$$

which is continuous function on $N$; if $\phi$ is the Abel method. $\mathscr{A}$, then the $\phi$ transform of $s$ is the continuous function on $I$ given by

$$
t(r)=(1-r) \sum_{n=0}^{\infty} s_{n} r^{n} \quad 0 \leqq r<1 .
$$

If $\phi$ is a regular and nonnegative summation method, then $\dot{\phi}$ is a functional of norm one on a closed subspace of $m_{0}$. Moreover if we denote the $\phi$-transform of $s$ by $t$ then $\lim \sup |t|$ is a semi-norm on $m_{0}$. Thus by the Hahn Banach theorem, the linear functional $\phi$ may be extended to a nonnegative linear functional on $m_{0}$ which satisfies

$$
|\underline{\phi}(s)| \leqq \lim \sup |t|,
$$

for each bounded sequence $s$; we shall denote this extension of $\dot{\phi}$ also by $\phi$; throughout the paper we will assume that $\phi$ has been extended to $m_{0}$ in such a way that (1) is fulfilled. Such an extension is never unique, and the results to be described hold for each such extension $\underline{\phi}:$

As a linear functional on $m_{0}, \underline{\phi}$ gives rise to a nonnegative measure on $\beta N$ which we also denote by $\phi$. Since $\phi$ is a regular summation method, the measure $\underline{\phi}$ is concentrated on $\underline{\beta} N-N$-we have $\int_{\underline{\underline{\beta} N}} d \underline{\phi}=1$. We shall write $\int s d \underline{\dot{\phi}}$ for $\int s^{[\underline{\beta}]} d \underline{\dot{\phi}}$.

Using (1) we can show

REMARK. If $s$ is a bounded sequence and $\underline{\phi}$ is a regular nonnegative summation method which is representable by either a summation matrix or a sequence-to-function transformation, then

$$
\lim \inf t \leqq \int_{\underline{\beta} N} s d \underline{\phi} \leqq \lim \sup t,
$$

where $t$ denotes the $\underline{\text {-transform }}$ of $s$.

The Abel summation method $\mathscr{A}$ induces translation-invariant measures on $\beta N$. This summation method will play a vital role in our discussion of Fourier transforms of sequences.

1. $L^{p}$ Spaces. If $p \geqq 1$ and $\phi$ is a regular summation method which is representable either by a summation matrix or by a sequence- 
to-function transformation, we define $L^{p}(\dot{\phi})$ as the space of sequences $s$ with the property that for each $\varepsilon>0$ there is a bounded sequence $s^{(s)}$ such that the sequence $\left|s-s^{(s)}\right|^{p}$ has a $\dot{\phi}$ transform whose limit superior is bounded in absolute value by $\varepsilon$; this definition is more restrictive than the usual definition of $L^{p}$ spaces. If $s$ is a sequence in an $L^{p}$ space we define

$$
\int_{\beta_{N}} s d \dot{\phi}=\lim _{s \rightarrow 0} \int_{\beta_{N}} s^{(s)} d \dot{\phi},
$$

where $\left\{s^{(\varepsilon)}\right\}$ is a set of bounded sequences which approximate $s$ in the sense that for each $\varepsilon>0$, there is a bounded sequence $s^{(\varepsilon)}$ such that the limit superior of the $\phi$-transform of $\left|s-s^{(\varepsilon)}\right|^{p}$ is less than $\varepsilon$ in absolute value. We norm $L^{p}$ by:

$$
\|s\|_{p}=\left(\int|s|^{p} d \phi\right)^{1 / p}=\lim _{\varepsilon \rightarrow 0}\left[\left.\int|s|^{(\varepsilon)}\right|^{p} d \phi\right]^{1 / p} .
$$

(Clearly the limit is independent of the choice of $\left\{s^{(\varepsilon)}\right\}$ ).

By Holder's inequality we have that for $1 \leqq q \leqq p, L^{p}(\phi) \subseteq L^{q}(\phi)$, and moreover $\|s\|_{q} \leqq\|s\|_{p}$.

As usual we identify two sequences $s$ and $t$ in $L^{p}(\phi)$ if

$$
\|s-t\|_{p}=0 \text {. }
$$

THEOREM. Let $\dot{\phi}$ be a regular nonnegative summation method and let $s$ be a sequence in $L^{p}(\phi), p \geqq 1$. Let $t$ denote the $\phi$-transform of $|s|^{p}$. Then

$$
\lim \inf t \leqq \int|s|^{p} d \dot{\phi} \leqq \lim \sup t<\infty .
$$

In particular if $\phi$ evaluates the sequence $\left|s_{n}\right|^{p}$ to $\sigma$, then

$$
\int|s|^{p} d \dot{\phi}=\sigma
$$

Proof. We deal only with the case where $\dot{\phi}$ is represented by a summation matrix $A=\left(a_{n k}\right)$ - the case where $\phi$ is representable by a sequence-to-function may be dealt with in a similar fashion. Let $s^{(\varepsilon)}$ be a set of bounded sequences approximating $s$, that is, for each $\varepsilon>0$ there is a bounded sequence $s^{(\varepsilon)}$ such that

$$
\lim \sup \sum_{k=0}^{\infty} a_{n k}\left|s_{k}-s_{k}^{(\varepsilon)}\right|^{p} \leqq \varepsilon
$$

If we take $\varepsilon=1$, 


$$
\begin{aligned}
\lim \sup & \sum_{h=1}^{\infty} a_{n k k}\left|s_{k}\right|^{p} \\
\leqq & 2^{p}\left[\lim \sup \sum_{k=n}^{\infty} a_{n k}\left|s_{k}^{\left({ }^{(s)}\right.}\right|^{p}\right. \\
& \left.+\lim \sup \sum_{k=n}^{\infty} a_{n k}\left|s_{k}-s_{k}^{(s)}\right|^{p}\right] \\
\leqq & 2^{p}\left[\lim \sup \sum a_{n k k}\left|s_{k}^{(s)}\right|^{p}+1\right] .
\end{aligned}
$$

Hence $\lim \sup |t|$ is finite.

Also

$$
\int|s|^{p} d A=\lim _{s \rightarrow 1)} \int\left|s^{(s)}\right|^{p} d A
$$

Since each $s^{(3)}$ is a bounded sequence.

$$
\begin{aligned}
& \lim \inf t_{n} \leqq \liminf \sum a_{n k}\left|s_{k}^{(s)}\right| p+C_{1} \varepsilon^{1 / p} \\
& \quad \leqq\left.\int\left|s^{(s)}\right|\right|^{p} d A+C_{1} \varepsilon^{1 / p} \\
& \leqq \lim \sup \sum_{k=0}^{\infty} a_{n k}\left|s^{(s)}\right|^{p}+C_{1} \varepsilon^{1 / p} \\
& \leqq \lim \sup t_{p}+C_{2} \varepsilon^{1 / p},
\end{aligned}
$$

where $C_{1}$ and $C_{2}$ are numbers not depending on $\varepsilon$. If we let $\varepsilon$ tend to zero we have the theorem.

Holder's inequality together with the technique of the above proof may be used to yield:

THEOREn. Let $r$ be a regular nonnegative summation method and let $s$ be a sequence in $L^{\prime \prime}(b) p \geqq 1$. If $t$ denoles the o-transform of so then

$$
\liminf t \leqq \int s d \dot{\lim \sup t}
$$

In particular if ovaluates s $10 \sigma$, then $\int_{\beta, 3} \operatorname{sdo}=\sigma$.

2. Fourier transforms. The Fourier transform $\hat{s}$ of a summation method $\dot{S}$ is defined as a functional on a space $M$ of test functions $f(\approx)=\sum_{n=0}^{\infty} \hat{f}(n) \tilde{z}^{n}$ analytic in the unit disc $D:|z|<1$, given by

$$
\begin{aligned}
& \hat{\phi}(f)=\int_{\hat{\underline{\beta}-1}-1}(\hat{f}(n)) \underline{\underline{\rho}} d \underline{\dot{\rho}} \\
& =\int_{\hat{z},} \hat{f}(n) d \underline{\dot{p}} \text {; }
\end{aligned}
$$

the Fourier transform $\hat{s}$ of a sequence $s=\left\{s_{n}\right\}$ is defined as the linear 
functional on $M$ given by

$$
\begin{aligned}
\hat{s}(f) & =\int_{\underline{\underline{\beta}} N} s^{\underline{\beta}}(\hat{f}(n)) \underline{\underline{\beta}} d \mathscr{\mathscr { C }}, \\
& =\int s_{n} \hat{f}(n) d \mathscr{\mathscr { A }}, \quad f \in M,
\end{aligned}
$$

where $\mathscr{L}$ is any measure on $\beta N-N$ induced by the Abel method.

The more customary definition of the Fourier transform, namely as the function of $[0,2 \pi]$ given by

$$
\int_{N} \exp (-i n \underline{\alpha}) s_{n} d \underline{\mathscr{A}}, \quad 0 \leqq \underline{\alpha}<2 \pi,
$$

is insufficient; S. P. Lloyd has given examples of sequences $s$ such that $\left|s_{n}\right|=1$ for all $\underline{\alpha}$ and such that $\int_{N} \exp (-i n \underline{\alpha}) s_{n} d \mathscr{A}$ vanishes for all $\underline{\alpha}$ cf [6]. Later we shall make some remarks about sequences $s$ which may be written

$$
s_{k}=\sum_{n} a_{n} \exp \left(i \underline{\alpha}_{n}^{\prime} k\right),
$$

where the Fourier coefficients $a_{n}$ are given by the formulas

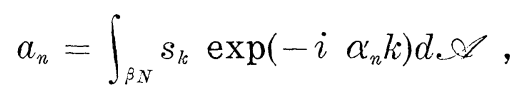

(that is, the sequence $s_{k} \exp (i \alpha k)$ is Abel summable for all $\alpha$ ), where each $\alpha_{n}$ is a number in $[0,2 \pi)$.

By $\mathrm{H}_{p}, p \geqq 1$ we understand the Hardy space of functions $f$ analytic in $D:|z|<1$ such that $\int_{0}^{2 z}\left|f\left(r e^{i \theta}\right)\right|^{p} d \theta$ is bounded for $0 \leqq$ $r<1$ [cf. 5 pp. 39].

THEOREM. If $L$ is a linear functional on a space of functions analytic in $D$ which agrees with the Fourier transform $\hat{\phi}$ of a regular summation method $\dot{\rho}$, then

$$
L(f)=0
$$

for each $f \in M$ which is also in $H_{1}$; also

$$
L(1 /(1-z))=1 \text {. }
$$

Proof. If $f \in H_{1}$ then $f(z)=\sum_{n=0}^{\infty} \hat{f}(n) z^{n},|z|<1$, and $\{\hat{f}(n)\}$ is a null sequence [cf. 5 pp. 70]. Since $\dot{\varphi}$ is a regular method, $\dot{\phi}$ must evaluate $\{\hat{f}(n)\}$ to zero. Hence $\hat{\phi}(f)=0$ for each $f \in H_{1} \cap M$. To establish (3) we simply note that since $\phi$ is regular, it must evaluate the sequence $\{1,1, \cdots\}$ to one, that is $\hat{\phi}(1 /(1-z))=1$. 
Our spaces of test functions will be (a) the space $M_{p}, p>1$, of functions

$$
f(z)=\sum_{n=0}^{\infty} \hat{f}(n) z^{n}
$$

analytic in $D$, such that

$$
\|f\|_{M_{p}}=\lim _{r \rightarrow 1-} \sup (1-r)^{1 / p^{\prime}}\left[\int_{0}^{2 \pi}\left|f\left(r^{1 / p^{\prime}} \exp i \theta\right)\right|^{p} d \theta / 2 \pi\right]^{1 / p}
$$

is finite-throut the paper the symbol $p^{\prime}$ denotes the number $p /(p-1)$ : Two functions $f, g$ are identified in $M_{p}$ in case

$$
\begin{aligned}
& (1-r)^{p / p^{\prime}} \int_{0}^{2 \pi} \mid f\left(r^{1 / p^{\prime}} \exp i \theta\right) \\
& \quad-\left.g\left({ }^{1{ }^{\prime}{ }_{p}^{\prime}} \exp i \theta\right)\right|^{p} d \theta
\end{aligned}
$$

tends to zero as $r$ tends to one. We norm each space $M_{p}$ by \|\|$_{M_{p}}$, (b) the space of functions

$$
f(z)=\sum_{n=0}^{\infty} \hat{f}(n) z^{n}
$$

such that

$$
\|f\|_{M_{\infty}}=\lim _{r \rightarrow 1-} \sup (1-r)|f(r \exp i \theta)|
$$

is finite. We identify two functions $f$ and $g$ in $M_{\infty}$ in case

$$
(1-r)|f(r \exp i \theta)-g(r \exp i \theta)|
$$

tends to zero as $r$ tends to 1 . We norm $M_{\infty}$ by \|\|$_{M_{\infty}}$. For $1<p<q<\infty$ we have $M_{p} \subseteq M_{q}$ of [3 pp. 623-625].

A linear functional $L$ on a normed space $M$ will be said to be welldefined if $L(f)=L(g)$ whenever $\|f-g\|=0, f, g \in M$.

For $p>0$ a sequence $s$ will be said to be strongly Abel-p-summable to $\sigma$ if

$$
\lim _{r \rightarrow 1}(1-r) \sum_{n=0}\left|s_{n}-\sigma\right|^{p} r^{n}=0 .
$$

The method of strong Abel-p-summability is regular for $p>0$.

Theorem. If $2 \leqq p<\infty$, and $L$ is a well-defined linear functional on $M_{p}$ such that

$$
L(1 / 1-z)=1,
$$

then there is a summation method $\phi$ which includes strong Abel-p'summability such that 


$$
\hat{\phi}(f)=L(f) \quad f \in M_{p}
$$

Proof. We define a summation method $\phi$ by $\int_{\beta_{N}} s d \phi=L(S)$, where $S(z)=\sum_{n=0}^{\infty} s_{n} z^{n}$, whenever the right hand is defined. If $f \in M_{p}$, then $L(f)$ is defined and $\hat{\phi}(f)=\int_{N} \hat{f}(n) d \phi=L(f)$. Now let $\left\{s_{n}\right\}$ be strongly Abel- $p^{\prime}$-summable to $\sigma$. Then $(1-r) \sum\left|s_{n}-\sigma\right|^{p^{\prime}} r^{n} \rightarrow 0$. Since $\sum\left(s_{n}-\sigma\right) z^{n}=S(z)-\sigma /(1-z)$ we have, by the Hausdorff-Young theorem cf [7, pp. 145], $(1-r) \int_{0}^{2 \pi}\left|S\left(r^{1 / p^{\prime}} e^{i \theta}\right)-\sigma /\left(1-r^{1 / p^{\prime}} e^{i \theta}\right)\right|^{p} d \theta \rightarrow 0$; thus $\|S-\sigma /(1-z)\|_{M_{p}}=0$. Since $L$ is well defined,

$$
L(S)=\sigma L(1 /(1-z))=\sigma
$$

by (4). Hence $\int_{N} s d \phi=\sigma$, that is, the method $\phi$ includes strongAbel- $p^{\prime}$-summability.

Similarly

THEOREM. If $L$ is a well defined linear functional on $M_{\infty}$ which satisfies (4), then there is a summation $\phi$ which includes strong-Abel1-summability such that $\hat{\phi}(f)=L(f), f \in M_{\infty}$.

If a summation matrix $A=\left(a_{n k}\right)$ has a sizable convergence field, then $\lim _{n \rightarrow \infty} \max _{k}\left|a_{n, k}\right|=0$; for example this condition must be satisfied if $A$ has the Borel property (cf [3]).

We denote by $\hat{A}$ the the Fourier transform of the summation method represented by the matrix $A$.

THEOREM. If $A=\left(a_{n k}\right)$ is a non-negative regular row-finite summation matrix such that $\lim _{n-\infty}$ l.u. $b_{k}\left|a_{n k}\right|=0, a_{n 0} \geqq a_{n 1} \geqq a_{n 2} \cdots$, then $\hat{A}\left(1 /\left(1-z e^{i \alpha}\right)=1\right.$ or 0 according as $\alpha$ is or is not congruent to zero modulo $2 \pi$.

Proof. We have $1 /\left(1-z e^{i \alpha}\right)=\sum_{n=0}^{\infty} e^{i n \alpha} z^{n}$. If $\alpha \equiv 0(\bmod 2 \pi)$, then $\hat{A}\left(1 /\left(1-z e^{i \alpha}\right)\right)=1$ by the regularity of $A$. If $\alpha \neq 0(\bmod 2 \pi)$, then since the sequence $\left\{a_{n k}\right\}$ is nonincreasing in $k$,

$$
\left|\sum_{k=0}^{\infty} a_{n k} e^{i k \alpha}\right| \leqq 8 a_{n 0} / \eta
$$

where $\eta$ is the distance of the point $\alpha$ from the multiples of $2 \pi$. Thus $A$ evaluates to zero each sequence $\left\{e^{i n \alpha}\right\}$ such that $\alpha$ is not a multiple of $2 \pi$, that is, $\hat{A}\left(1 /\left(1-z e^{i \alpha}\right)=0\right.$ if $\alpha \neq 0(\bmod 2 \pi)$. 
Theorem. Let $P$ denote the Norlund summation method, so that the P-transform of a sequence $s$ is the sequence $\left\{\sum_{h=0}^{\infty} p_{n-k} s_{k} / P_{n}\right\}$, where the numbers $p_{n}, P_{n}$ satisfy the conditions

$$
P_{n}=\sum_{k=0}^{n} p_{k}, \quad p_{k}=0(1), \quad P_{n} \rightarrow \infty .
$$

Then for almost all $\alpha$ in $[0,2 \pi)$

$$
\hat{P}(1 / 1-z \exp i \alpha)=0 .
$$

This result is proved in [1, pp. 325-326].

THEorem. If $s$ is a sequence in $L^{p}(\mathscr{A}), 1<p \leqq 2$, then $\hat{s}$ is a bounded functional on $M_{p}$, and

$$
\|\hat{s}\|^{p} \leqq \lim \sup (1-r) \sum_{n=0}^{\infty}\left|s_{n}\right|^{p}, r^{n}
$$

Proof. If $p \leqq 2$, then by the Hausdorff-Young theorem

$$
\begin{aligned}
& \left(\sum_{n=0}^{\infty}|\hat{f}(n)|^{p^{\prime}} r^{n}\right)^{1 / p^{\prime}} \\
& \quad \leqq\left[\int_{0}^{2 \pi} \mid f\left(\left.r^{1 / p^{\prime}} \exp (i \theta)\right|^{p} d \theta / 2 \pi\right]^{1 / p}, \quad f \in M_{p} .\right.
\end{aligned}
$$

Hence, if $s \in L^{p}(\mathscr{C})$, we have by Holder's inequality

$$
\begin{aligned}
|\hat{s}(f)| & \leqq I \int_{\beta N}\left\{s_{n} \hat{f}(n)\right\} d \mathscr{\mathscr { C }} \\
& \leqq \lim _{r \rightarrow 1-} \sup (1-r)\left(\left.\sum_{n=0}^{\infty}\left|s_{n}\right|\right|^{p} r^{n}\right)^{1 / p}\left(\sum_{n=0}^{\infty}|\hat{f}(n)|^{p^{\prime}} r^{n}\right)^{1 / p^{\prime}} \\
& \leqq\|f\|_{M_{p}} \lim \operatorname{sum}\left[(1-r)\left(\sum_{n=0}^{\infty}\left|s_{n}\right|^{p} \gamma^{n}\right)\right]^{1 / p} \cdot
\end{aligned}
$$

Since the last member is bounded, $\hat{s}$ is a bounded functional on $M_{p}$. If $s$ is a bounded sequence such that the sequence $\left\{\left|s_{n}\right|^{p}\right\}$ is Abel summable, then $\|\hat{s}\| \leqq\|s\|_{p}$ - when $\hat{s}$ is considered a linear functional on $M_{p}$.

Theorem. If $s$ is a sequence in $L^{p}(\mathscr{P}) 2 \leqq p<\infty$, then

$$
\|\hat{s}\| \geqq\|s\| / \lim \sup (1-r) \sum\left|s_{n}\right|{ }^{p} r^{n},
$$

when $\hat{s}$ is considered a functional on $M_{p}$, provided that the sequence $\left\{\left|s_{n}\right|^{p}\right\}$ is not Abel summable to zero. If the sequence $\left\{\left|s_{n}\right|^{p}\right\}$ is Abel summable, then $\|\hat{s}\| \geqq\|s\|$. If $\hat{s}(f)=0$ for all $f \in M_{p}$, then $\|s\|_{p}=0$.

Proof: We let 


$$
\begin{aligned}
\hat{f}(n) & =\left|s_{n}\right|^{p-2} \overline{s_{n}} & & \text { if } s_{n} \neq 0, \\
& =0 & & \text { if } s_{n}=0 .
\end{aligned}
$$

If follows from the Hausdorff Young theorem that $f^{\prime}(z)=\sum \hat{f}(n) z^{n} \in M_{p}$, and

$$
\begin{aligned}
\left\|f^{\prime}\right\|_{u_{p}} & \leqq \lim \sup \left[(1-r) \sum|\hat{f}(n)|^{p^{\prime}} r^{n}\right]^{1 / p^{\prime}} \\
& =\lim \sup \left[\left.(1-r) \sum_{n=0}^{\infty}\left|s_{n}\right|\right|^{p} r^{n}\right]^{1 / p^{\prime}} .
\end{aligned}
$$

Hence if $\|f\|_{M_{p}} \neq 0$,

$$
\begin{aligned}
\|\hat{s}\| & \geqq|\hat{s}(f)| /\|f\|_{\text {M促 }} \\
& \geqq\|s\|_{p}^{p} / \lim \sup \left[(1-r) \sum\left|s_{n}\right|^{p} r^{n}\right]^{1 / p^{\prime}} .
\end{aligned}
$$

If the sequence $\left\{\left|s_{n}\right|^{p}\right\}$ is Abel summable to a nonzero value,

$$
\|\hat{s}\| \geqq\|s\|_{p}{ }^{p} /\|s\|_{p}{ }^{p / p^{\prime}}=\|s\|_{p} \text {. }
$$

If $\hat{s}$ annihilates $M_{p}$ it must annihilate the function $f$ defined above, and thus $\|s\|_{p}=0$.

We make a few remarks about the sequence $s$ which may be written as exponential series

$$
s_{k}=\sum_{n=0}^{\infty} a_{n} \exp \left(i \alpha_{n} k\right) \quad k=0,1, \cdots,
$$

where the numbers $\alpha_{n}$ lie in the interval $[0,2 \pi)$ and the numbers $a_{n}$ are given by the formulas

$$
\begin{aligned}
a_{n} & =\int_{\beta N} s_{k} \exp \left(-i \alpha_{n} k\right) d \cdot \Omega \\
& =\lim _{r=1-}(1-r) \sum_{n=0}^{\infty} s_{k} \exp \left(-i \alpha_{n} k\right) r^{k} \quad n=0,1, \cdots,
\end{aligned}
$$

(we assume that the sequence $\left\{s_{k} \exp (i \alpha k)\right\}$ is Abel summable for each $\alpha$ in $[0,2 \pi))$. We also have

$$
a_{n}=\hat{s}\left(1 / 1-z \exp \left(-i \alpha_{n}\right)\right) .
$$

We have the following version of the Riesz Fisher theorem:

Theorem. If $\sum\left|a_{p}\right|^{2}<\infty$, then the Fourier transforms of the exponential polynomials

$$
s_{k}^{(j)}=\sum_{n=i}^{j} a_{n} \exp \left(i \alpha_{n} l i\right), \quad j=1,2, \cdots,
$$

converge to a bounded linear functional $\sigma$ on $M_{2}$, in the sense that 


$$
\lim _{j \rightarrow \infty}\left\|\sigma-\hat{\boldsymbol{s}}^{(j)}\right\|=0
$$

and

$$
\|\sigma\|^{2}=\sum_{n=1}^{\infty}\left|a_{n}\right|^{2}=\lim _{j=\infty}\left\|\hat{s}^{(j)}\right\|_{2}^{2},
$$

when each $\hat{s}^{(j)}$ is considered a functional on $M_{2}$.

Proof. Let $f(z)=\sum \hat{f}(n) z^{n}$ be a function in $M_{2}$. Then

$$
\begin{aligned}
& \left|\hat{s}^{\left(j^{\prime}\right)}(f)-\hat{s}^{\left(j^{\prime \prime}\right)}(f)\right| \\
& \quad=\int_{\beta_{N}}\left(\sum_{J^{\prime}}^{j^{\prime \prime}} a_{n} \exp \left(i \alpha_{n} k\right)\right) \hat{f}(k) d \underline{\mathscr{\mathscr { A }}} \\
& \quad \leqq\left(\int_{\beta_{N}}\left|\sum_{j^{\prime}}^{j^{\prime \prime}} a_{n} \exp \left(i \alpha_{n} k\right)\right|^{2} d \underline{\mathscr{A}}\right)^{1 / 2}\|f\|_{M_{2}} \\
& \quad \leqq\left(\sum_{n=j^{\prime}}^{j^{\prime \prime}}\left|a_{n}\right|^{2}\right)^{1 / 2}\|f\|_{M_{2}},
\end{aligned}
$$

which tends to zero as $j^{\prime}$ and $j^{\prime \prime}$ tend to infinity, where the above integration is carried out with respect to $k$. Therefore, for each $f \in M_{2}$ the sequence $\left\{\hat{s}^{(j)}(f)\right\}$ is a Cauchy sequence of numbers and hence converges. Let $\sigma(f)=\lim \widehat{s}^{(j)}(f)$. It is readily verified that $\sigma(f)$ depends linearly on $f$. Also

$$
\begin{aligned}
|\sigma(f)| & =\left|\lim \hat{s}^{(j)}(f)\right| \\
& \leqq\left(\sum_{n=0}^{j}\left|a_{n}\right|^{2}\right)^{1 / 2}\|f\|_{M_{2}} ;
\end{aligned}
$$

hence if we regard $\sigma$ as a functional on $M_{2},\|\sigma\|<\left(\sum\left|a_{j}\right|^{2}\right)^{1 / 2}$. If we take

$$
f(z)=\sum \hat{f}(k) z^{k}
$$

where

$$
\widehat{f}(k)=\sum_{n=0}^{j} a_{n} \exp \left(-i \alpha_{n} k\right),
$$

then the sequence $\{|\hat{f}(k)|\}^{2}$ is Abel summable to $\sum_{n=1}^{j}\left|a_{n}\right|^{2}$; thus

$$
\left.\int_{\beta_{N}}\left|\hat{f}(k)^{2} d \underline{\mathscr{A}}=\|f\|_{M_{2}^{2}}=\sum_{n=1}^{j}\right| a_{n}\right|^{2} \text {. }
$$

Since $s^{(j)}(f)=\sum\left|a_{n}\right|^{2},\left\|\hat{s}^{(j)}\right\|^{2}=\sum_{n=1}^{j}\left|a_{n}\right|^{2}$. Since $\|\sigma\|=\lim _{j \rightarrow \infty}\left\|\hat{s}^{(j)}\right\|$, $\|\sigma\|^{2}=\sum_{n=1}^{\infty}\left|a_{n}\right|^{2}$. 


\section{REFERENCES}

1. N. Bary, A treatise on trigonometric series, Vol. 2, Pergamon Press, New York, 1964.

2. L. Gillman and M. Jerison, Rings of continuous functions, Van Nostrand, Princeton, New Jersey, 1960.

3. G. Hardy and J. E. Littlewood, A convergence criterion of Fourier series, Math. Z., 28 (1928), 612-634.

4. J. D. Hill, Summation of sequences of 0's and 1's, Math. Ann., (2), 46 (1945), 556-562.

5. K. M. Hoffman, Banach spaces of analytic functions, Prentice Hall, Englewood Cliffs, New Jersey, 1962.

6. S. P. Lloyd, On the $L^{2}$ space of a Banach limit, Proc. Amer. Math. Soc., 20 (1969), 429-433.

7. A Zygmund, Trigonometric series, Second edition, New York Chelsea, 1952.

Received August 6, 1970. This research was supported by N. S. F. Grant 7686.

UNIVERSITY OF MINNESOTA 



\section{PACIFIC JOURNAL OF MATHEMATICS}

\section{EDITORS}

H. SAMELSON

Stanford University

Stanford, California 94305

C. R. HOBBY

University of Washington

Seattle, Washington 98105
J. DugundJI

Department of Mathematics

University of Southern California

Los Angeles, California 90007

RICHARD ARENS

University of California

Los Angeles, California 90024

\section{ASSOCIATE EDITORS}

E. F. BeCKENBACH

B. H. NeumanN

F. WOLF

K. YosHIDA

\section{SUPPORTING INSTITUTIONS}

UNIVERSITY OF BRITISH COLUMBIA

CALIFORNIA INSTITUTE OF TECHNOLOGY

UNIVERSITY OF CALIFORNIA

MONTANA STATE UNIVERSITY

UNIVERSITY OF NEVADA

NEW MEXICO STATE UNIVERSITY

OREGON STATE UNIVERSITY

UNIVERSITY OF OREGON

OSARA UNIVERSITY
UNIVERSITY OF SOUTHERN CALIFORNIA STANFORD UNIVERSITY

UNIVERSITY OF TOKYO

UNIVERSITY OF UTAH

WASHINGTON STATE UNIVERSITY

UNIVERSITY OF WASHINGTON

AMERICAN MATHEMATICAL SOCIETY

NAVAL WEAPONS CENTER

Printed in Japan by International Academic Printing Co., Ltd., Tokyo, Japan 


\section{Pacific Journal of Mathematics}

\section{Vol. 40, No. $1 \quad$ September, 1972}

Alex Bacopoulos and Athanassios G. Kartsatos, On polynomials

approximating the solutions of nonlinear differential equations........

Monte Boisen and Max Dean Larsen, Prüfer and valuation rings with zero

divisors ..........................................

James J. Bowe, Neat homomorphisms

David W. Boyd and Hershy Kisilevsky, The Diophantine equation

$$
u(u+1)(u+2)(u+3)=v(v+1)(v+2) \ldots \ldots \ldots \ldots \ldots \ldots \ldots
$$

George Ulrich Brauer, Summability and Fourier analysis ...............

Robin B. S. Brooks, On removing coincidences of two maps when only one,

rather than both, of them may be deformed by a homotopy ............

Frank Castagna and Geert Caleb Ernst Prins, Every generalized Petersen

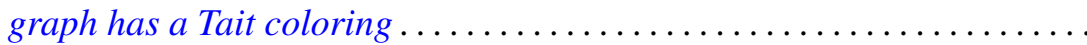

Micheal Neal Dyer, Rational homology and Whitehead products ..........

John Fuelberth and Mark Lawrence Teply, The singular submodule of a

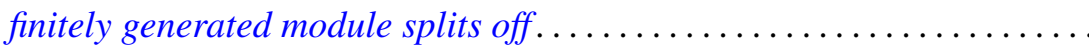

Robert Gold, $\Gamma$-extensions of imaginary quadratic fields ............ 83

Myron Goldberg and John W. Moon, Cycles in k-strong tournaments.......

Darald Joe Hartfiel and J. W. Spellmann, Diagonal similarity of irreducible

matrices to row stochastic matrices...............

Wayland M. Hubbart, Some results on blocks over local fields ..

Alan Loeb Kostinsky, Projective lattices and bounded homomorphisms....

Kenneth O. Leland, Maximum modulus theorems for algebras of operator

valued functions ...

Jerome Irving Malitz and William Nelson Reinhardt, Maximal models in the

language with quantifier "there exist uncountably many" ..

John Douglas Moore, Isometric immersions of space forms in space

forms.

Ronald C. Mullin and Ralph Gordon Stanton, A map-theoretic approach to

Davenport-Schinzel sequences ....................

Chull Park, On Fredholm transformations in Yeh-Wiener space. .

Stanley Poreda, Complex Chebyshev alterations ..............

Ray C. Shiflett, Extreme Markov operators and the orbits of Ryff. ...

Robert L. Snider, Lattices of radicals .....................

Ralph Richard Summerhill, Unknotting cones in the topological

category ................................

Charles Irvin Vinsonhaler, A note on two generalizations of $\mathrm{QF}-3 \ldots \ldots 229$

William Patterson Wardlaw, Defining relations for certain integrally

parameterized Chevalley groups...................

William Jennings Wickless, Abelian groups which admit only nilpotent

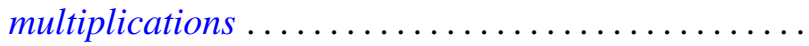

